\title{
Analysis and Measurement of Multiple Antenna Systems for Fading Channel Prediction in Moving Relays
}

\author{
N. Jamaly ${ }^{1}$, R. Apelfröjd ${ }^{2}$, A. Belen Martinez ${ }^{3}$, M. Grieger ${ }^{3}$, T. Svensson ${ }^{1}$, M. Sternad ${ }^{2}$, G. Fettweis ${ }^{3}$ \\ ${ }^{1}$ Department of Signals and Systems, Chalmers University of Technology, Gothenburg, Sweden \\ ${ }^{2}$ Department of Engineering Sciences, Signals and Systems, Uppsala University, Sweden \\ ${ }^{3}$ Vodafone Chair Mobile Communications Systems, Technische Universität Dresden, Germany
}

\begin{abstract}
The performance of wireless data transmission to mobile vehicles is improved if channel state information is available at the transmitter but movement of vehicles causes outdating of channel estimates. The concept of a predictor antenna has recently been proposed, where an antenna is placed in front of other antennas on the roof of the vehicle to sense the radio environment in advance. This can comparatively provide an order-of-magnitude improvement in channel prediction performance. A potential problem with this idea is that closely placed antennas will experience mutual electromagnetic couplings. These may reduce the efficiency of the predictor antenna concept if they are not taken into account. In this paper, we discuss about how to treat the forgoing issue and eventually evaluate a promising candidate on measured channels. We argue that only open-circuit voltage method would be realistic for the present application. The usefulness of the proposed decoupling method is demonstrated on field measurements obtained in downtown Dresden, Germany. We also partly address the sensitivity of the open-circuit decoupling method to the accuracy of the utilized network parameters.
\end{abstract}

Index Terms-Multi-element antennas, channel state prediction, moving relays, multipath measurement.

\section{INTRODUCTION}

In modern wireless communication systems, the presence of channel state information (CSI) at the transmitter enables link adaptation, multi-user scheduling, beamforming, spatial multiplexing, space division multiple access, and coordinated multipoint transmission. Delays in the feedback control loop cause outdating of the CSI when transmitting to a moving vehicle. This problem becomes increasingly severe with larger delays, higher vehicle velocities, and shorter carrier wavelengths. Prediction based on past received channel estimates by Kalman or Wiener prediction can to some extent alleviate the problem [1]. However, these methods become inadequate in multipath fading environments when the required prediction horizon in time corresponds to a movement of more than 0.3 carrier wavelengths in space. For high mobility users, this limitation prevents accurate CSI to be obtained in the current 3GPP Long Term Evolution (LTE) systems with carrier frequencies exceeding $1 \mathrm{GHz}$ [2].

Recently, the concept of a predictor antenna system, using a multi-element antenna on the vehicle, has been proposed to overcome this problem [2]. In this system at least two antennas

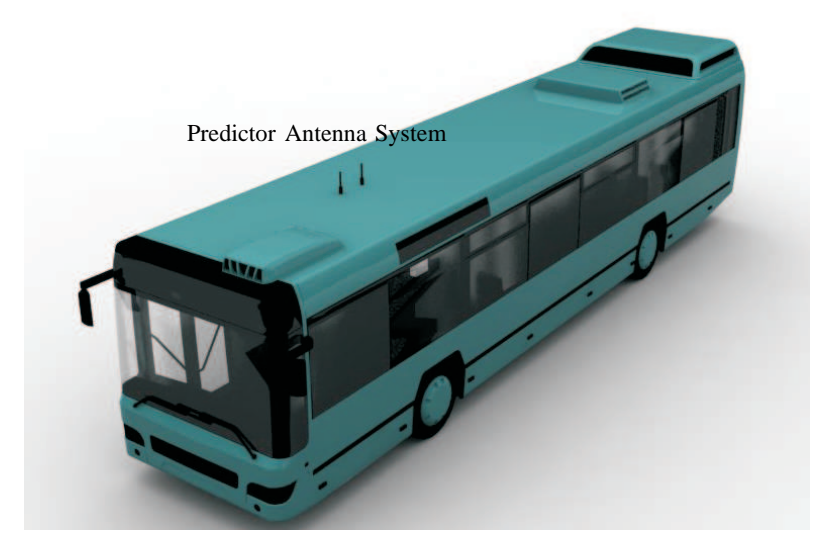

Fig. 1. Predictor antenna system installed on a bus.

are used: a predictor antenna, located in the direction of travel, and -at least- one receiver antenna behind it, see Fig. 1. The role of the predictor antenna in a Frequency-Division Duplexing (FDD) system is to constantly measure the CSI and feed it back to the transmitter. In a Time-Division Duplexing (TDD) system, the predictor sends the uplink pilots for channel estimation at the base station. Provided that the system is moving on a straight line, the receiver antenna always arrives at the same position where the predictor antenna was a few moments earlier. Thus, the channel estimates from the predictor antenna can be used as CSI predictions for efficient communication with the receiver antenna. The temporal crosscorrelation between appropriately delayed signals at the ports of the two antennas is then a suitable measure of CSI prediction accuracy.

This type of system, which is especially valuable in public transportation systems due to the combination of their high velocity and high data demand, was proposed and experimentally investigated in [2]. The present paper extends and improves the predictor antenna concept in two respects. First, we experimentally investigate the use of two monopole antennas over a flat ground plane by field measurements using an orthogonal frequency-division multiplexing (OFDM) link with $20 \mathrm{MHz}$ bandwidth. Compared to the preliminary 
investigations in [2], which used two dipole antennas on a rather cluttered vehicle roof, the results in this paper show a much higher peak antenna correlation and thus a far better CSI prediction accuracy. Moreover, we propose and evaluate the use of a simple method for the compensation of coupling effects between antenna elements. This increases the design flexibility of sets of antennas or antenna arrays on vehicles that are used as predictor antennas. Throughout this paper, one has to clearly distinguish between the received signals' temporal cross-correlation and the more known spatial correlation between them.

There are numerous studies that tackle the mutual coupling problem in multi-antenna systems (see for instance [3] and references therein). The common view in the literature is that decoupling techniques relying on the input network parameters, e.g., open-circuit voltage method, S-parameter method etc., are not sufficiently precise in practice since the receiving and transmitting properties of multi-element antennas are not in general the same [4]. In contrast, more accurate yet elaborate methods, like the receiving mutual impedance method which is commonly used in direction of arrival estimation applications, are not applicable for coupling removal in multipath fading environments due to high computational complexity.

In the predictor antenna framework, here we suggest using the open-circuit voltage method. We claim that by restricting ourselves to a particular class of multi-element antennas, this implies no loss in accuracy. This proposal is evaluated based on field measurements.

\section{Array Antenna Coupling Compensation: A REVIEW}

Coupling compensation has long been a challenge in compact multi-element antenna arrays. The effectiveness of the available methods in the literature depends on the type of the antenna used and its applications. In general, among these decoupling methods, the ones which are potentially useful in the framework of this paper can be grouped into two major categories. The first category may include the open-circuit voltage method, the S-parameter method, and the (isolated) element pattern method [3]. The nature of the foregoing methods is the same, and the primary advantage of them is their simplicity. The common concern regarding these methods is that the Thevenin (or Norton) equivalent circuits used to characterize the transmitting antennas' radiation behavior is not generally sufficient to hold also in the receive mode.

In contrast, the second category includes the full-wave method, the calibration method, and the receiving mutual impedance method. Although fairly elaborated in comparison with those in the former category, these methods are generally more accurate. Perhaps the full-wave method which is commonly based on the method of moments is the most accurate, yet computationally inefficient. In the calibration method, a coupling matrix has to be either iteratively measured or calculated, which is a tedious task [5]. In addition to these, there comes the receiving mutual impedance method, which is fairly accurate and efficient for direction finding purposes [3]. However, in this method, the receiving mutual impedance matrix should first be determined which is, in turn, a function of angle of arrival for the incoming waves [6].

Nevertheless, when it comes to receiving antennas in a multipath environment, since in general the scattering characteristics of the antennas and consequently their coupling properties are some functions of the angle of arrival, the methods in the second category require huge computational resources or measurements for the coupling matrices or the receiving mutual impedances. This drawback makes them practically inefficient if not useless for the aforementioned purposes. Therefore, for antennas in multipath scenarios, we need to resort to a method from the first category.

\section{Open-circuit Voltage Decoupling Method}

The goal in this section is to briefly review the open-circuit voltage decoupling method. This method plays the major role in the current paper wherein we underline its important application for compact antennas in multipath scenarios. We first highlight the limitation in application of this method and later revisit the formula available in the literature for its realisation.

\section{A. Limitations in Open-Circuit Voltage Method}

In principle, the Thevenin equivalent circuit for current driven antennas ${ }^{1}$, which is widely used for the characterization of the antennas in the transmit mode, has a limited usage for antennas in the receive mode [7]. For instance, in the receive mode, the total scattered power created by an incident wave upon the antenna structure is the sum of the reradiated power predicted by an equivalent circuit and the scattered power while the antenna is open-circuited (or short-circuited if the Norton model is used) [8]. Note that the equivalent circuit does not predict the latter power. Therefore, it cannot cover the total radiation mechanism which is necessary to characterize the impact of coupling in the receive mode. However, for a certain class of array antennas, known as minimum scattering antennas, wherein the open-circuit scattered field in the presence of an incident wave is zero, the radiation mechanism in the receive mode and the transmit mode are similar and expressible in terms of the equivalent circuits [9]. Under this constraint, the open-circuit voltage method for coupling compensation turns out to be quite precise. Recall that, based on the discussion presented before, the latter method is likely the most efficient method for coupling compensation in a multipath environment.

Fortunately, many lossless single-mode antennas approximate minimum scattering antennas [10]. Therefore, to be able to design a well-behaved predictor antenna system in which the coupling can be compensated effectively, we need to use some single-mode and fairly lossless antennas. Let us denote the input impedance matrix of an $n$-element antenna system by $\mathbf{Z}_{n \times n}$. As a rule of thumb, to assess whether an antenna array

\footnotetext{
${ }^{1}$ Or equivalently the Norton equivalent circuit for voltage driven antennas.
} 


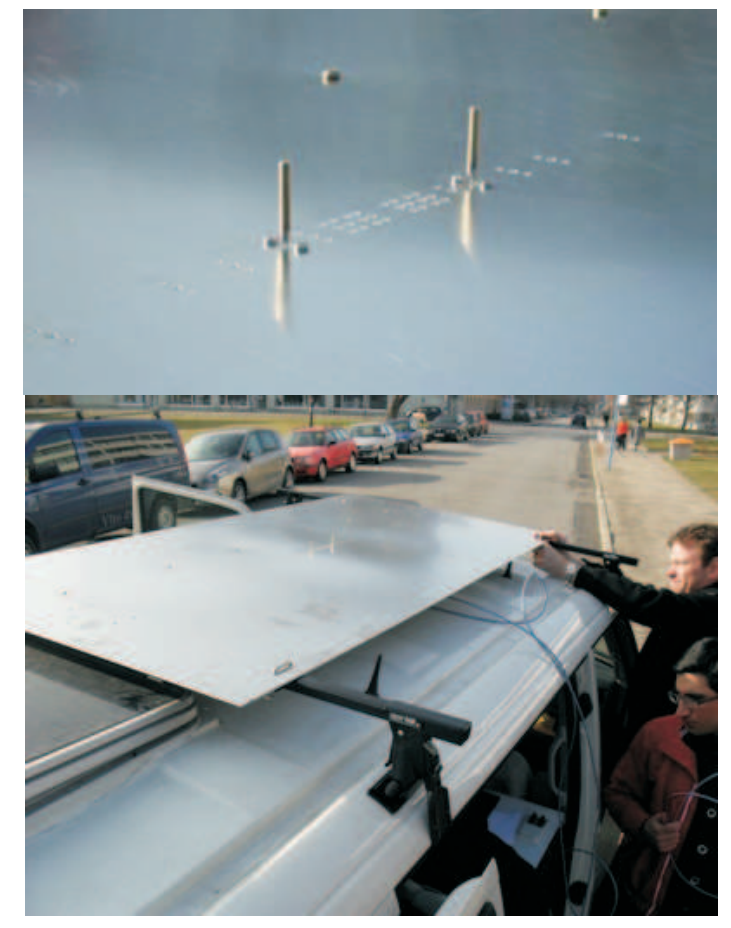

Fig. 2. Two in-line monopoles over a fairly large aluminium plane which were used in our measurement campaign $(f=2.68 \mathrm{GHz})$.

can approximate minimum scattering antennas, one needs to check if the pattern overlap matrix, $\mathbf{C}$, in ohms reads [11][12]

$$
\mathbf{C} \approx \Re[\mathbf{Z}]
$$

wherein the function $\Re$ returns the real part of its argument and $\mathbf{C}$ is defined as

$$
\mathbf{C}=\frac{1}{\eta} \oint_{4 \pi} \mathbf{G}^{\dagger}(\Omega) \cdot \mathbf{G}(\Omega) d \Omega .
$$

Here, $\mathbf{G}_{2 \times n}(\Omega)$ is the matrix of open-circuit embedded patterns of this multi-element antenna system whose rows are its corresponding vertical $\theta$ polarization and horizontal $\psi$ polarization components. The symbol $\Omega$ signifies the solid angle. In [13], reverberation chambers have been proposed for a quick measurement of the pattern overlap matrix.

\section{B. Open-circuit Voltage Method}

To find a general formula for the use of this method, we can follow similar steps in deriving the relation between the opencircuit $\mathbf{C}_{\circ}(f, t)$ and terminated $\mathbf{C}_{r}(f, t)$ covariance matrices of the received baseband signals $v_{r}(f, t)$ at different ports as detailed in [14, Chapter 4]. The result is:

$$
\mathbf{C}_{\circ}=2\left(\mathbf{Z}+\mathbf{Z}_{r}\right) \mathbf{Z}_{r}^{-1} \Re\left[\mathbf{Z}_{r}\right]^{\frac{1}{2}} \mathbf{C}_{r} \Re\left[\mathbf{Z}_{r}\right]^{\frac{1}{2}} \mathbf{Z}_{r}^{-1^{\dagger}}\left(\mathbf{Z}+\mathbf{Z}_{r}\right)^{\dagger}
$$

wherein the terminating impedances (or receiver impedances) were cast in a diagonal matrix $\mathbf{Z}_{r}$ and the dagger superscript ${ }^{\dagger}$ signifies the conjugate transpose. Note that all the network parameters in (2) are functions of frequency, nevertheless, the arguments are dropped for the sake of conciseness. The above expression merits further discussion. It is the covariance of the received voltage signals created by the same random incoming waves in an open-circuit circumstance. We reiterate that many lossless single-mode antennas approximate minimum scattering antennas [10]. For a minimum scattering antenna, the elements which are left open-circuited do not perturb the embedded patterns of other nearby radiation elements. This can be interpreted as if there is no coupling among them. Thus, the expression at left hand side of the above equation gives the received signals' covariance matrix at these $n$ ports as if there would be no coupling among the antenna elements. Coupling compensation can also be directly performed on baseband OFDM signals by

$$
\bar{v}_{\circ}(f, t)=\left(\mathbf{Z}(f)+\mathbf{Z}_{r}(f)\right) \mathbf{Z}_{r}(f)^{-1} \bar{v}_{r}(f, t)
$$

where $\bar{v}_{r}$ is the $n$-entry column vector of received baseband antenna signals at subcarrier $f$ and $\bar{v}_{\circ}$ is the corresponding vector of compensated signals. In the next section, we apply the foregoing formula to measured data to removed the impact of coupling.

\section{Measurement CAMpaign}

This section describes the measurements conducted to verify both the notion of using predictor antenna system for moving relays in multipath fading environment and the benefits of removing coupling in the foregoing system. Perhaps the only reliable method for evaluating the practicability of an ambitious communication scheme is field trials for experimenting with true physical channels including the analog front-end [15]. The measurements were performed in spring 2012 in an urban environment in downtown Dresden, Germany. A downlink OFDM system with a single antenna base station located at the top of a $55 \mathrm{~m}$ high building was used. The base station antenna was $+45^{\circ}$ cross-polarized (KATHREIN 80010541), with a $9^{\circ}$ down tilt, a beamwidth of $58^{\circ}$, and a gain of $18 \mathrm{dBi}$. A Volkswagen T4 (VW) with two in-line thin $\lambda / 4$ monopoles antennas installed on its roof was used as the moving relay. To avoid refraction and abnormal reflection and scattering from the nonuniform roof, we used a large aluminum plane sheet as shown in Fig. 2.

Measurements were conducted at a velocity of approximately $50 \mathrm{~km} / \mathrm{h}$ for several antenna separations in two different multipath scenarios: line-of-sight (LOS) and non-line-of-sight (NLOS). The base station transmited only known signals, referred to as pilots. These were transmitted over 2048 subcarriers spread over a bandwidth of $20 \mathrm{MHz}$ at a carrier frequency of $f=2.68 \mathrm{GHz}$ for every $\tau=0.1 \mathrm{~ms}$. The complex baseband channel response, $h(f, t)$, was calculated in frequency domain as

$$
h(f, t)=\frac{v_{r}(f, t)}{v_{p}(f, t)}
$$

where $v_{p}(f, t)$ denotes the pilot signals at the subcarrier frequency $f$ and the time $t=\tau, 2 \tau, \ldots, N \cdot \tau$, with $N$ being the number of measured OFDM symbols. Due to some filtering 


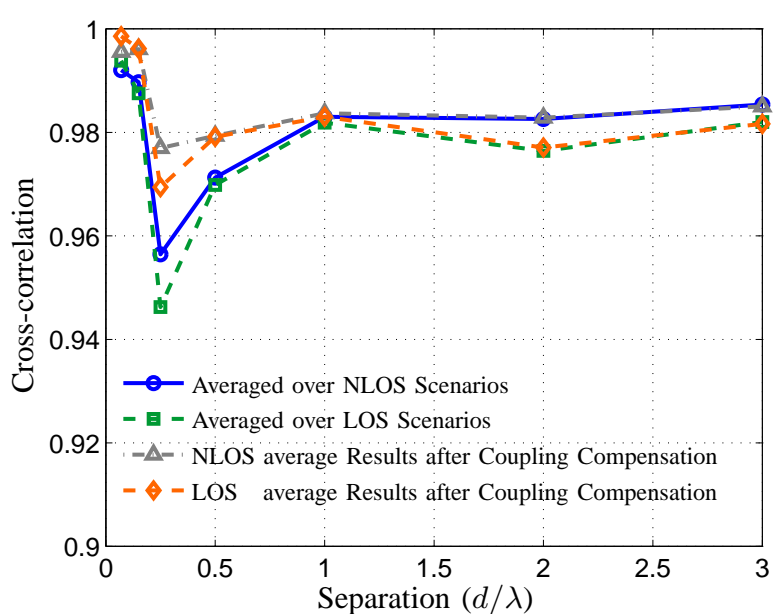

(a) Mean Cross-correlation

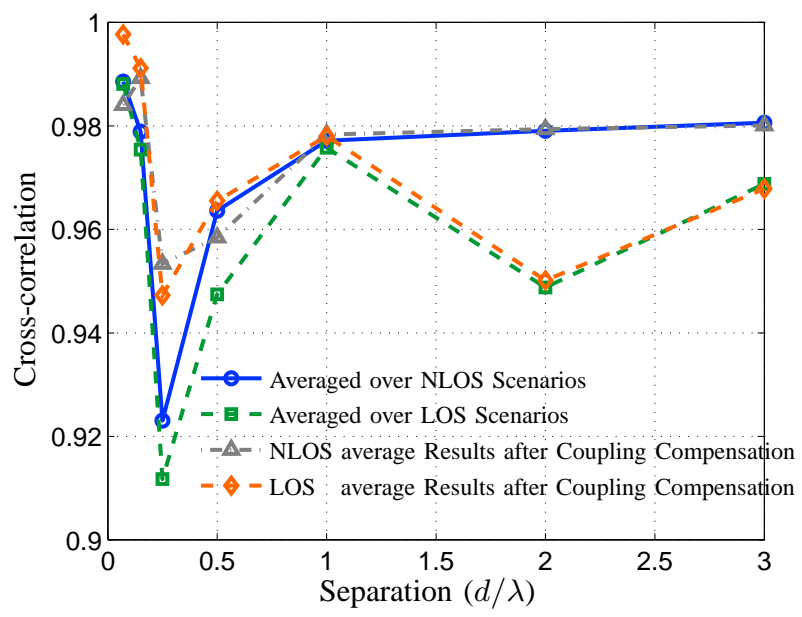

(b) $5 \%$ Cross-correlation

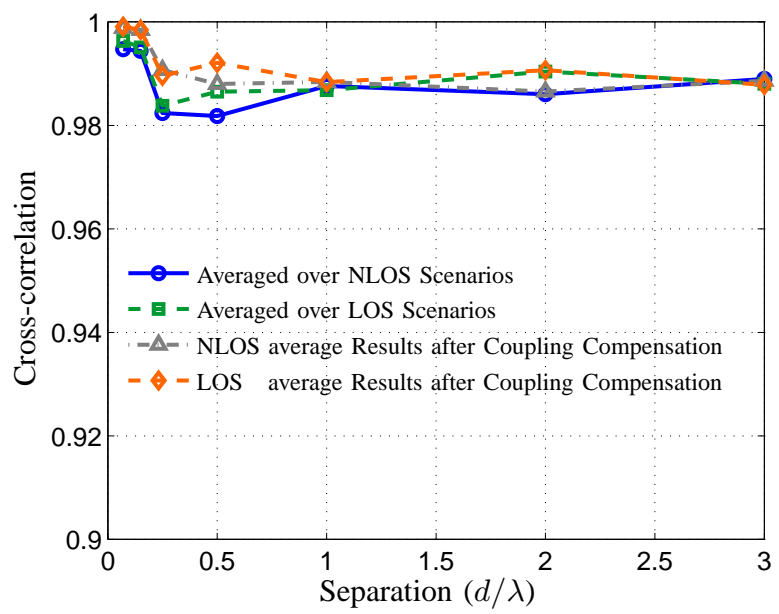

(c) $95 \%$ Cross-correlation

Fig. 3. Measurement Results: Cross-correlations between the received signals in line-of-sight (LOS) and non-line-of-sight (NLOS) scenarios. effects in the receive equipment, the frequency response over the first and last subcarriers is inaccurate. Therefore, only 1960 subcarriers are used in current analysis. For each location and antenna separation, three sets of measurements, each of length 0.5 s (i.e., $N=5000$ ) were stored. Note that, the network parameters and radiation characteristic of the antennas over the whole bandwidth are fairly the same. Thus, the same parameters were used for coupling compensation for all the subcarrier signals.

\section{AnAlysis of the Results}

As the main criterion, the temporal cross-correlation between the measured signals at the ports of the predictor antenna and the receiver antenna was calculated both in the presence and after compensation of the antenna coupling. Different subcarriers experience different fading scenarios and thus demonstrate different cross-correlations. Therefore, we have a range of correlations associated with these subcarriers. The cross-correlation of unity indicates an ideally reliable channel prediction. The outcome of our measurements and processing of the results are illustrated in Figs. 3. In these figures the mean, $5 \%$ - and $95 \%$-percentiles of the cross-correlations over all subcarriers versus separation between the predictor and the receiver antennas are shown. Recall that the coupling compensation -or coupling removal- was conducted based on the discussion presented in the preceding section. In almost all cases, the coupling removal enhanced the corresponding crosscorrelation. The enhancement for monopole antennas extends from a few percents up to $10 \%$. The maximum increase is at separations shorter than $d<\lambda$, which is indeed expected. The presented results are also averaged over three repetitions of the measurements for each scenario.

Further study of Equation (2) reveals that the accuracy of coupling compensation also depends on accuracy of the impedance matrices used in this equation. This becomes particularly crucial for separations wherein the self or mutual impedances are subject to sudden changes in their resistive or reactive parts. Therefore, it is worth briefly studying dependence of the coupling compensation on the mutual impedances which by its own is an indicator of coupling. To do so, for the antennas under test, we focused on $d=0.5 \lambda$ element separation. Our numerical full wave simulation based on the method of moments shows that the mutual resistance and reactance for the quarter wavelength monopoles around the foregoing separation vary within the range $\pm 12 \Omega$. For the time being, the changes in self-impedances are neglected to focus on the variation of cross-correlation versus changes in mutual impedances. This presumption may not be realistic as we shall see in a moment, nevertheless, it provides an instructive and fair understanding regarding dependence of coupling removal on mutual impedances.

Fig. 4 illustrates contours of mean cross-correlation based on the measurement data versus slight changes of the mutual impedance. Note that in practice, these slight changes may originate from inaccuracy in the locations of the antennas 


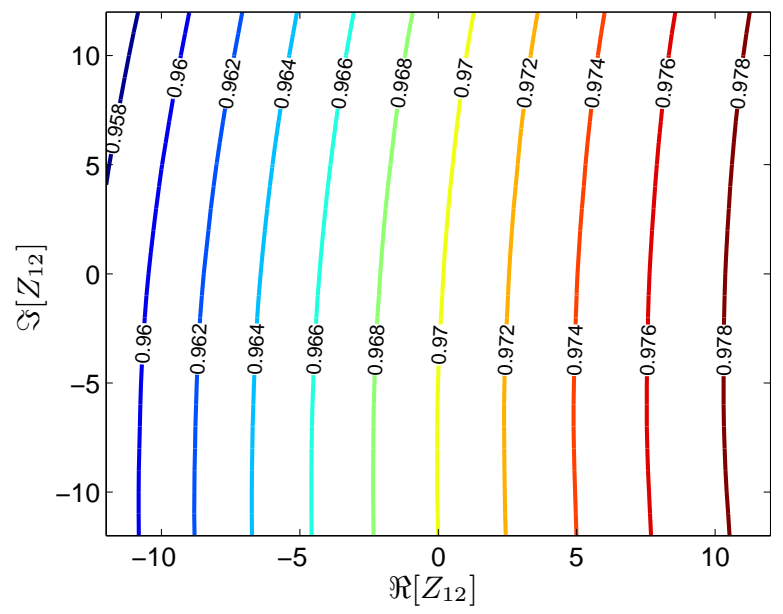

Fig. 4. Contours of mean cross-correlation (NLOS) versus the variation in the mutual impedance for $d=0.5 \lambda$. $\Re$ and $\Im$ denote the real and imaginary components of their arguments.

during installation, small differences between frequency of operation and the resonance frequency of the antennas, or from coupling and leakage through connectors etc. Fairly similar observations are made when ones studies $5 \%$ and $95 \%$ cross-correlations. Based on this figure, the best mutual impedance rendering the optimum decoupling for the desired cross-correlation is $Z_{12}=12 \Omega$. However, in reality the mutual impedance used in Fig. 3 is $Z_{12}=-3-j 12 \Omega$. The distinction between the two foregoing impedances can best be attributed to omitting the changes in self-impedances in calculation of the contours presented in Fig. 4. In conclusion, since for this specific measurement, the overall cross-correlations are already near one, a slight inaccuracy in measuring the impedance matrix could lead to deteriorated results after coupling compensation. Hence, having an accurate impedance matrix for coupling removal in moving relays is significant.

In summary, the high cross-correlation in the measurements shows how effective the proposed prediction system is. Comparing the current results with those of [2], in general, demonstrates that the monopoles prove to be far better options than the dipoles in this application. This is attributed to the associated radiation patterns in the elevation plane.

\section{CONCLUSION}

In this paper, we first argued that different precise decoupling techniques well known in the literature are not suitable options for applications in multipath environments due to their computational burden. We thus reasoned that we have to resort to the classic simple methods based on the input network parameters, which impose some restriction in choices of antennas. We demonstrated the efficiency of the predictor antenna system and the usefulness of the proposed coupling compensation in an actual scenario by virtue of a thorough measurement campaign in downtown Dresden, Germany. Firstly, the results illustrated the effectiveness of predictor antenna system in fading channel prediction for moving relays. In particular, the results verified that using quarter-wavelength monopoles instead of half-wavelength dipoles as used in [2] was naturally advantageous. Furthermore, we illustrated that for shorter separations between the predictor and the receiver antennas coupling compensation based on input network parameters was beneficial. The compensation based on the proposed method depended on the accuracy of the input network parameters. We presented a brief numerical study to reveal sensitivity of the foregoing method with respect to the mutual impedances. In the latter short study, we neglected the impact of selfimpedances which remains for the future work.

\section{ACKNOWLEDGMENT}

The authors acknowledge S.-E. Breuer, E. Ohlmer, and I. Wiklund for their assistance in the measurement campaign.

\section{REFERENCES}

[1] D. Aronsson, Channel Estimation and Prediction for MIMO OFDM Systems: Key Design and Performance Aspects of Kalman-based Algorithms. Uppsala University, March 2011.

[2] M. Sternad, M. Grieger, R. Apelfrojd, T. Svensson, D. Aronsson, and A. Martinez, "Using "predictor antennas" for long-range prediction of fast fading for moving relays," in IEEE Wireless Communications and Networking Conference Workshops (WCNCW), April 2012.

[3] H. T. Hui, "Decoupling methods for the mutual coupling effects in antenna arrays: A review," Recent Patents on Engineering, vol. 1, pp. 187-193, 2007.

[4] H. Hui, "Compensating for the mutual coupling effect in direction finding based on a new calculation method for mutual impedance," IEEE Antennas and Wireless Propagation Letters, vol. 2, no. 1, pp. 26-29, 2003.

[5] H. Aumann, A. Fenn, and F. Willwerth, "Phased array antenna calibration and pattern prediction using mutual coupling measurements," IEEE Transactions on Antennas and Propagation, vol. 37, no. 7, pp. 844-850, 1989.

[6] H. Hui, "A new definition of mutual impedance for application in dipole receiving antenna arrays," IEEE Antennas and Wireless Propagation Letters, vol. 3, no. 1, pp. 364-367, 2004.

[7] A. Love, "Comment on the equivalent circuit of a receiving antenna," IEEE Antennas and Propagation Magazine, vol. 44, no. 5, pp. 124-125, 2002.

[8] R. Collin, "Limitations of the thevenin and norton equivalent circuits for a receiving antenna," IEEE Antennas and Propagation Magazine, vol. 45, no. 2, pp. 119-124, 2003.

[9] W. Kahn and H. Kurss, "Minimum-scattering antennas," IEEE Transactions on Antennas and Propagation, vol. 13, no. 5, pp. 671-675, September 1965.

[10] R. C. Hansen, Phased Array Antennas, ser. Microwave and Optical Engineering, K. Chang, Ed. John Wiley \& Sons, 1998.

[11] N. Jamaly, A. Derneryd, and Y. Rahmat-Samii, "A revisit to spatial correlation in terms of input network parameters," IEEE Antennas and Wireless Propagation Letters, vol. 11, pp. 1342-1345, 2012.

[12] W. Wasylkiwskyj and W. Kahn, "Theory of mutual coupling among minimum-scattering antennas," IEEE Transactions on Antennas and Propagation, vol. 18, no. 2, pp. 204-216, March 1970.

[13] N. Jamaly and A. Derneryd, "Fast measurement of antenna pattern overlap matrix in reverberation chamber," Electronics Letters, vol. 49, no. 5, pp. 318-319, 2013.

[14] N. Jamaly, "Multiport Antenna Systems for Space-Time Wireless Communication," Chalmers University of Technology, Sweden, Tech. Rep., March 2013. [Online]. Available: http://publications.lib.chalmers.se/records/fulltext/174702/174702.pdf

[15] S. Caban, J. Garcia Naya, and M. Rupp, "Measuring the physical layer performance of wireless communication systems: Part 33 in a series of tutorials on instrumentation and measurement," IEEE Instrumentation Measurement Magazine, vol. 14, no. 5, pp. 8-17, 2011. 